14,15

\title{
Диффузионные процессы в свободно подвешенных смектических пленках
}

\author{
(c) I. Śliwa ${ }^{1}$, A.B. Захаров ${ }^{2, \text { q }}$ \\ ${ }^{1}$ Institute of Molecular Physics, Polish Academy of Sciences, \\ Poznaǹ, Poland \\ ${ }^{2}$ Институт проблем машиноведения РАН, \\ Санкт-Петербург, Россия \\ IE-mail: alexandre.zakharov@yahoo.ca
}

(Поступила в Редакцию 31 января 2017 г.)

\begin{abstract}
Предложена молекулярная модель, описывающая трансляционную диффузию в свободно подвешенных смектических пленках (СПСП) в воздухе. Эта модель основана на теории случайных блужданий и позволяет рассчитать коэффициент трансляционной диффузии (КТД), направленный поперек смектических слоев (вдоль направления директора). Все необходимые для расчета КТД величины были получены в рамках обобщенной среднеполевой модели, учитывающей не только анизотропные взаимодействия между ближайшими соседями молекул, образующих СПСП, но и стабилизирующее влияние границы раздела смектик/воздух. Возникающая при этом пространственная неоднородность параметров порядка по сечению СПСП ведет к тому, что поверхностное натяжение на границе смектик/воздух не только подавляет термофлуктуации в приповерхностных слоях, но и полностью подавляет трансляционную диффузию молекул из СПСП в воздух. Результаты расчета размерной трансляционной диффузии в объеме СПСП, образованной молекулами 5-n-алкил-2-(4-n-(перфлуороалкил-метиленеокси))пентила, в процессе ее утоньшения показали, что величина КТД монотонно возрастает по мере утоньшения смектической пленки.
\end{abstract}

Работа выполнена при поддержке РФФИ (грант № 16-02-00041a).

DOI: $10.21883 /$ FTT.2017.08.44768.27

\section{1. Введение}

Одной отличительной особенностью, которой обладают смектические жидкие кристаллы (СЖК), является то, что они при определенных термодинамических условиях способны образовывать свободно подвешенные смектические пленки (СПСП) [1,2]. Эти СПСП представляют собой уникальные образцы устойчивых квазидвумерных систем в трехмерных пространствах, образующих слоистую структуру, в которой длинные оси молекул в каждом смектическом слое флуктуируют относительно нормали к этим слоям. При этом толщина каждого слоя $d$ порядка длины ЖК-молекулы, а вся толщина СПСП $L=N d$ кратна толщине единичного смектического слоя. Экспериментально с помощью оптических и калориметрических методов было показано, что по мере нагревания $N$-слойной СПСП происходит выдавливание одного или нескольких ЖК-слоев в мениск, который поддерживает стабильное состояние смектической пленки [1]. При этом было показано, что в процессе нагревания 25-слойной СПСП, образованной молекулами 5-n-алкил-2-(4-n-(перфлуороалкилметиленеокси)) пентила (H10F5MOPР), при температуре выше температуры фазового перехода смектикA (SmA)-изотропное (I) состояние в объеме ЖК-фазы $\left(T_{A I}(\mathrm{~b})\right)$ начинается процесс послойного выдавливания внутренних слоев пленки в мениск. Сначала выдавливается 10 слоев, затем 4, и далее по одному слою, заканчивая двуслойной СПСП, которая рвется при температуре $T_{A I}(N=2) \sim 385 \mathrm{~K}$, что на $\sim 27 \mathrm{~K}$ выше тем- пературы $T_{A I}(\mathrm{~b}) \sim 358 \mathrm{~K}$. При этом реализуется следующая последовательность послойного утоньшения СПСП: $25 \rightarrow 15 \rightarrow 11 \rightarrow 10 \rightarrow \ldots \rightarrow 2[1]$. Оптические измерения показали, что по мере роста температуры процессу выдавливания определенного числа слоев из квазидвумерной $(2 D)$ СПСП предшествует процесс внутреннего разупорядочения одного или нескольких смектических слоев, что выражается в резком уменьшении величин как ориентационных, так и трансляционных параметров порядка (ПП), соответствующих выдавливаемым слоям. Такой механизм плавления $2 D$ СПСП, когда разупорядочение начинается с внутренних слоев пленки и распространяется к ее границам, противоположен традиционному процессу плавления трехмерных (3D) упорядоченных систем, таких как кристаллы или металлы, в которых с ростом температуры разупорядочение начинается с поверхности твердого тела [3]. В таких низкоразмерных системах плавление заменяется серией переходов, при которых толщина пленки уменьшается на один или несколько смектических слоев. По сути дела, роль температуры плавления $N$-слойной СПСП играет температура $T_{A I}(N)$, при которой начинается процесс послойного выдавливания в мениск одного или нескольких слоев ЖК-материала, а само число $N$ становится дискретным параметром $2 D$-системы, уменьшение которого позволяет СПСП оставаться устойчивой и трансляционно упорядоченной. Обе поверхности СПСП, граничащие с воздухом, представляют собой физические границы раздела двух сред (смектик/воздух), а поверхностное натяжение этих границ подавляет термофлуктуации мо- 
лекул в приповерхностных смектических слоях. Возникающая при этом пространственная неоднородность ПП по сечению СПСП ведет к существенным изменениям структурных и термодинамических характеристик этих пленок по сравнению с объемными образцами $[1,2,4-6]$. Следует ожидать, что эта неоднородность также оказывает влияние и на диффузионные процессы в тонких смектических пленках. Диффузия в таких анизотропных системах, как жидкие кристаллы (ЖК) описывается тензором трансляционной диффузии (ТТД) [7] $D_{i j}$, где $i, j=x, y, z$ - индексы, соответствующие осям координат. Посредством выбора системы координат ТТД может быть преобразован к диагональной форме с двумя основными элементами $D_{z z}=\mathscr{D}_{\|}$и $D_{x x}=D_{y y}=\mathscr{D}_{\perp}$, соответствующими коэффициентам трансляционной диффузии (КТД) в направлении вдоль и поперек направления директора $\hat{\mathbf{n}}$. В случае объемной SmA фазы процесс трансляционной диффузии вдоль направления директора n подразумевает скачок диффундирующей молекулы через потенциальный барьер $\Phi$, возникающий между слоями смектической фазы. В системе координат, где ось $z$ совпадает с направлением $\hat{\mathbf{n}}$, потенциал $\Phi(z)$ является периодической функцией пространственной переменной z с периодом $d: \Phi(z+d)=\Phi(z)$, а КТД может быть записан в виде [8]

$$
\mathscr{D}_{\|}=D_{z z}=\frac{\left\langle z^{2}\right\rangle}{2 \tau}
$$

где $\left\langle z^{2}\right\rangle$ - среднеквадратичное смещение диффундирующей молекулы, а $\tau$ - время, необходимое молекуле для того, чтобы совершить перескок через барьер $\Phi(z)$ с выбранного смектического слоя на соседний слой SmA фазы. Поскольку вероятность перескока молекулы „вверх ${ }^{6}$ и „вниз“ одинакова, то КТД необходимо разделить на два.

Целью этой статьи является исследование структурных и диффузионных свойств свободно подвешенных смектических пленок в воздухе и помещенных во внешнее электрическое поле $\mathbf{E}$, направленное поперек смектических слоев. Поскольку в процессе послойного утоньшения СПСП, вызванного ростом температуры $T>T_{A I}(\mathrm{~b})$, наблюдается сильная пространственная неоднородность как трансляционных, так и ориентационных ПП по сечению смектической пленки [4,9], то это должно способствовать аномальному поведению КТД $\mathscr{D}_{\|}$в направлении, совпадающим с направлением директора $\hat{\mathbf{n}}$. Этот диффузионный процесс будет исследован в рамках молекулярной модели диффузии, основанной на теории случайных блужданий [10], а все необходимые для вычисления параметры будут рассчитаны с помощью модели [4], в рамках которой процесс послойного утоньшения объясняется разупорядочением нескольких внутренних слоев СПСП, при сохранении устойчивого упорядочения в приповерхностных слоях вплоть до момента разрыва двуслойной смектической пленки.

\section{2. Молекулярная модель для описания трансляционной диффузии}

Предположим, что СПСП соединена с двух концов с мениском, в который в процессе утоньшения смектической пленки происходит сброс выдавливаемых поверхностными силами (или расклинивающим давлением) [9] разупорядоченных изотропных слоев ЖК-материала. Рассмотрим в первом приближении, что мениск оказывает слабое влияние на термодинамическое состояние $N$-слойной смектической пленки. Таким образом, будем рассматривать только $N$ дискретных взаимодействующих друг с другом смектических слоев, подвергнутых со стороны ограничивающих поверхностей ЖК/воздух стабилизирующему воздействию. Будем считать, что каждый слой имеет толщину $d$ порядка длины ЖК-молекулы. При этом будем предполагать, что $N$-слойная СПСП, образованная $M=\sum_{i=1}^{N} N_{i}$ молекулами и занимающая объем $V$, подвержена воздействию внешнего электрического поля $\mathbf{E}$, направленного поперек смектических слоев. Здесь $N_{i}$-число молекул в $i$-ом слое. Будем предполагать, что молекулы $i$-го слоя взаимодействуют только между собой и молекулами двух соседних слоев: верхнего $(i+1)$ и нижнего $(i-1)$.

Согласно молекулярной модели трансляционной диффузии, основанной на теории случайных блужданий [10], трансляционная диффузия поперек смектических слоев имеет место, когда молекула совершает прыжок с $(i+1)$-го на $i$-ый слой. Здесь отсчет слоев ведется от границы раздела ЖК/воздух к центру смектической пленки. В результате КТД может быть записан в виде [8]

$$
\mathscr{D}_{i, i+1}=\mathscr{D}_{\|}^{i, i+1}=\frac{\left\langle z_{i, i+1}^{2}\right\rangle}{\tau_{i, i+1}},
$$

где $\left\langle z_{i, i+1}^{2}\right\rangle=\left\langle z^{2}\right\rangle=\int_{0}^{d} z^{2} h(z) d z-$ среднеквадратичное смещение диффундирующей молекулы, $h(z)$ - одночастичная функция распределения соответствующая $(i+1)$-ому слою [4], а $\tau_{i, i+1}-$ время, необходимое молекуле для перескока с $(i+1)$-го слоя на $i$-слой. Таким образом, мы исследуем трансляционную диффузию из объема СПСП в сторону раздела ЖК/воздух. В нашем случае время $\tau_{i, i+1}$ может быть записано в виде

$$
\tau_{i, i+1}=\tau_{0} \exp \left[\frac{\Delta \Phi_{i, i+1}}{k_{B} T}\right],
$$

где $\tau_{0}$ - время осцилляции молекулы около положения равновесия в объеме смектической пленки, $k_{B}$ - постоянная Больцмана, $T$ - абсолютная температура, а

$$
\Delta \Phi_{i, i+1}=\Phi_{i}(\max )-\Phi_{i+1}(\min )
$$

величина потенциального барьера разделяющего $(i+1)$ и $i$-смектические слои. Здесь $\Phi_{i}(\max )$ и $\Phi_{i+1}(\min )-$ величины, соответствующие максимальному и минимальному значениям потенциала $\Phi(z)$ в $i$ - и $(i+1)$-слоях.

Таким образом, для того чтобы рассчитать КТД $\mathscr{D}_{i, i+1}$, нам прежде всего необходимо рассчитать величины 
$\Phi_{i}(\max )$ и $\Phi_{i+1}(\min )$, а также одночастичную функцию распределения $h(z)$, соответствующую $(i+1)$-смектическому слою.

Поскольку мы рассматриваем $N$-слойную смектическую пленку при фиксированной температуре $T$ и объеме $V$ в качестве устойчивой термодинамической системы, характеризующейся гомеотропной ориентацией директора на границе раздела ЖК/воздух, то можно предположить, что эффективное стабилизирующее взаимодействие ЖК-молекул, принадлежащих двум граничным слоям $(i=1$ и $i=N)$, сильнее, чем взаимодействие ЖК-молекул в объеме СПСП. Это позволяет нам ввести характерный параметр $W_{0}$, ответственный за стабилизирующее влияние границ раздела ЖК/воздух, величина которого больше величины параметра $V_{0}\left(W_{0}>V_{0}\right)$ ответственного за взаимодействие ЖК-молекул в объеме СПСП [4]. В рамках этого подхода можно ввести $N$ анизотропных эффективных потенциалов $\Phi_{i}(i=1, \ldots, N)$, соответствующих каждому смектическому слою $i[4]$

$$
\begin{gathered}
-\frac{3}{V_{0}} \Phi_{1}\left(z_{1}, \theta_{1}\right)=\left[\frac{W_{0}}{V_{0}} q_{1}+q_{2}+\Delta\left(q_{1}+\frac{1}{2}\right)\right. \\
\left.+\alpha \cos \left(\frac{2 \pi z_{1}}{d}\right)\left(\frac{W_{0}}{V_{0}} \sigma_{1}+\sigma_{2}\right)\right] P_{2}\left(\cos \theta_{1}\right) \\
-\frac{3}{V_{0}} \Phi_{1<i<N}\left(z_{i}, \theta_{i}\right)=\left[\sum_{j=i-1}^{i+1} q_{j}+\Delta\left(q_{i}+\frac{1}{2}\right)\right. \\
\left.+\alpha \cos \left(\frac{2 \pi z_{i}}{d}\right) \sum_{j=i-1}^{i+1} \sigma_{j}\right] P_{2}\left(\cos \theta_{i}\right) \\
-\frac{3}{V_{0}} \Phi_{N}\left(z_{N}, \theta_{N}\right)=\left[\frac{W_{0}}{V_{0}} q_{N}+q_{N-1}+\Delta\left(q_{N}+\frac{1}{2}\right)\right. \\
\left.+\alpha \cos \left(\frac{2 \pi z_{N}}{d}\right)\left(\frac{W_{0}}{V_{0}} \sigma_{N}+\sigma_{N-1}\right)\right] P_{2}\left(\cos \theta_{N}\right)
\end{gathered}
$$

где $z_{i}-$ расстояние вдоль оси $z$, направленное вдоль нормали к $i$-ому смектическому слою, $\theta_{i}-$ полярный угол, образованный длинной осью молекулы и осью $z, P_{2}\left(\cos \theta_{i}\right)$ - полином Лежандра второго порядка, $V_{0}$ - энергетический параметр системы, связанный с температурой перехода SmAI и определяющий температурную шкалу модели, $W_{0}$ - параметр системы, соответствующий эффективному стабилизирующему поверхностному взаимодействию, $\alpha=2 \exp \left[-\left(\frac{\pi r_{0}}{d}\right)^{2}\right]$ - еще один параметр системы, характеризующий длину алкилных „хвостов“ ЖК-молекул, изменяющийся в пределах $0 \leq \alpha \leq 2$ [11]. Здесь $r_{0}$ - характеристическая длина, ассоциирующаяся с ядром ЖК-молекулы. В нашем случае силой, ответственной за устойчивое упорядочение в приповерхностных слоях является дополнительное ЖК/воздух парное взаимодействие $\frac{W_{0}-V_{0}}{3}\left[q_{1}+\alpha \cos \left(\frac{2 \pi z_{1}}{d}\right) \sigma_{1}\right]$ и $\frac{W_{0}-V_{0}}{3}\left[q_{N}+\alpha \cos \left(\frac{2 \pi z_{N}}{d}\right) \sigma_{N}\right], \quad$ в $\quad$ то время как безраз- мерный параметр $\Delta=\frac{\epsilon_{0} \epsilon_{a v} E^{2}}{V_{0}}$ ответственен за воздействие электрического поля $\mathbf{E}$ на смектическую пленку, где $\epsilon_{0}-$ диэлектрическая проницаемость вакуума, $\epsilon_{a}$ - диэлектрическая постоянная ЖК-системы, а $v=V / M$ - объем, приходящийся на одну молекулу смектической пленки. Следует отметить, что вклад электрического поля $\mathbf{E}$ в величины эффективных потенциалов $\Phi_{i}(i=1, \ldots, N)$ равен $-\left\langle\frac{\epsilon_{0} \epsilon_{a}}{2}(\mathbf{E} \cdot \hat{\mathbf{n}})^{2}\right\rangle=-\frac{\epsilon_{0} \epsilon_{a} E^{2}}{2}\left\langle\cos ^{2} \theta_{i}\right\rangle=-\frac{\epsilon_{0} \epsilon_{a} E^{2}}{3}\left(q_{i}+\frac{1}{2}\right)$.

Оба ПП, ориентационный $q_{i}$ и трансляционный $\sigma_{i}$, соответствующие $i$-ому слою, удовлетворяют системе нелинейных интегральных уравнений [4]

$$
q_{i}=\left\langle P_{2}\left(\cos \theta_{i}\right)\right\rangle_{i},
$$

и

$$
\sigma_{i}=\left\langle\cos \left(\frac{2 \pi z_{i}}{d}\right) P_{2}\left(\cos \theta_{i}\right)\right\rangle_{i}
$$

Здесь $\langle(\ldots)\rangle_{i}-$ среднее статистико-механическое, усредненное с помощью одночастичной функции распределения, соответствующей $i$-ому слою

$$
h_{i}\left(z_{i}, \theta_{i}\right)=\mathscr{A}_{i}^{-1} \exp \left[-\frac{\Phi_{i}}{k_{\mathrm{B}} T}\right],
$$

a $\mathscr{A}_{i}$ - нормировочная постоянная. Набор ПП $q_{i}$ и $\sigma_{i}$, соответствующих $i$-ому смектическому слою в $N$-слойной СПСП, может быть получен решением системы $2 N$ нелинейных интегральных уравнений $(6)-(8)$ при фиксированном числе слоев $N$, температуре $T$ и трех параметрах системы: $\alpha, W_{0} / V_{0}$ и $\Delta$.

Располагая набором ПП $q_{i}$ и $\sigma_{i}(i=1, \ldots, N)$ мы можем рассчитать эффективный потенциал, необходимый для вычисления потенциального барьера $\Delta \Phi_{i, i+1}$, соответствующего $i$-слою

$$
\Phi_{i}\left(z_{i}\right)=\int_{-1}^{+1} d\left(\cos \theta_{i}\right) \Phi_{i}\left(z_{i}, \theta_{i}\right),
$$

который в нашем случае принимает вид

$$
\begin{array}{r}
-\frac{3}{V_{0}} \Phi_{1}\left(z_{1}\right)=\frac{W_{0}}{V_{0}} q_{1}+q_{2}+\Delta\left(q_{1}+\frac{1}{2}\right) \\
+\alpha \cos \left(2 \pi z_{1}\right)\left(\frac{W_{0}}{V_{0}} \sigma_{1}+\sigma_{2}\right), \\
-\frac{3}{V_{0}} \Phi_{1<i<N}\left(z_{i}\right)=\sum_{j=i-1}^{i+1} q_{j}+\Delta\left(q_{i}+\frac{1}{2}\right) \\
+\alpha \cos \left(2 \pi z_{i}\right) \sum_{j=i-1}^{i+1} \sigma_{j}, \\
-\frac{3}{V_{0}} \Phi_{N}\left(z_{N}\right)=\frac{W_{0}}{V_{0}} q_{N}+q_{N-1}+\Delta\left(q_{N}+\frac{1}{2}\right) \\
+\alpha \cos \left(2 \pi z_{N}\right)\left(\frac{W_{0}}{V_{0}} \sigma_{N}+\sigma_{N-1}\right) .
\end{array}
$$

Здесь и в дальнейших вычислениях $\bar{z}_{i}=z_{i} / d$ является безразмерной пространственной переменной, которая 
изменяется в пределах от 0 и до 1 , а в целях упрощения последующих записей верхняя черта над переменной $z$ опущена. Принимая во внимание характер взаимодействия молекул, образующих смектическую пленку, выражения для потенциального барьера принимают вид

$$
\begin{gathered}
-\frac{3 \Delta \Phi_{1,2}}{V_{0}}=-\frac{3}{V_{0}}\left(\Phi_{1}(\max )-\Phi_{2}(\min )\right) \\
=\frac{W_{0}}{V_{0}} q_{1}+q_{2}+\alpha\left(\frac{W_{0}}{V_{0}} \sigma_{1}+\sigma_{2}\right)+\Delta\left(q_{1}-q_{2}\right) \\
\quad-q_{1}-q_{2}-q_{3}+\alpha\left(\sigma_{1}+\sigma_{2}+\sigma_{3}\right) \\
-\frac{3 \Delta \Phi_{i, i+1}}{V_{0}}=-\frac{3}{V_{0}}\left(\Phi_{i}(\max )-\Phi_{i+1}(\min )\right) \\
=q_{i+1}+q_{i}+q_{i-1}+\alpha\left(\sigma_{i+1}+\sigma_{i}+\sigma_{i-1}\right)+\Delta\left(q_{i}-q_{i+1}\right) \\
-q_{i}-q_{i+1}-q_{i+2}+\alpha\left(\sigma_{i}+\sigma_{i+1}+\sigma_{i+2}\right) \\
-\frac{3 \Delta \Phi_{N, N-1}}{V_{0}}=\frac{3}{V_{0}}\left(\Phi_{N}(\max )-\Phi_{N-1}(\min )\right) \\
=\frac{W_{0}}{V_{0}} q_{N}+q_{N-1}+\alpha\left(\frac{W_{0}}{V_{0}} \sigma_{N}+\sigma_{N-1}\right)+\Delta\left(q_{N-1}-q_{N}\right) \\
-q_{N}-q_{N-1}-q_{N-2}+\alpha\left(\sigma_{N}+\sigma_{N-1}+\sigma_{N-2}\right)
\end{gathered}
$$

В свою очередь, величина среднеквадратичного смещения диффундирующей молекулы из $(i+1)$-слоя в $i$-ый равна

$$
\left\langle z_{i, i+1}^{2}\right\rangle=\left\langle z^{2}\right\rangle=\int_{0}^{1} z^{2} h(z) d z,
$$

а функция $h(z)$ имеет вид

$$
h\left(z_{i}\right)=\frac{\int_{-1}^{1} d\left(\cos \theta_{i}\right) h\left(z_{i}, \theta_{i}\right.}{\int_{(i-1)}^{i} d z_{i} \int_{-1}^{1} d\left(\cos \theta_{i}\right) h\left(z_{i}, \theta_{i}\right)} .
$$

\section{3. Результаты вычислений структурных и диффузионных свойств смектических пленок}

Принимая во внимание результаты экспериментальных исследований, проведенных с 25-слойной частично флуорированной пленкой H10F5MOPP [1], величина параметра $\mathrm{N}$ была выбрана равной 25. Согласно теории Мак-Миллана [11], фазовый переход SmA-I осуществляется при $\alpha>0.98$, и, следовательно, наш выбор $\alpha=1.05$ вполне согласуется с последним ограничением. Что касается выбора параметра $W_{0} / V_{0}$, то мы руководствовались тем фактом, что параметризация $W_{0} / V_{0}=5$ позволила достаточно точно описать поведение теплоемкости $C_{v}$ в процессе утоньшения 25-слойной смектической пленки, образованной молекулами
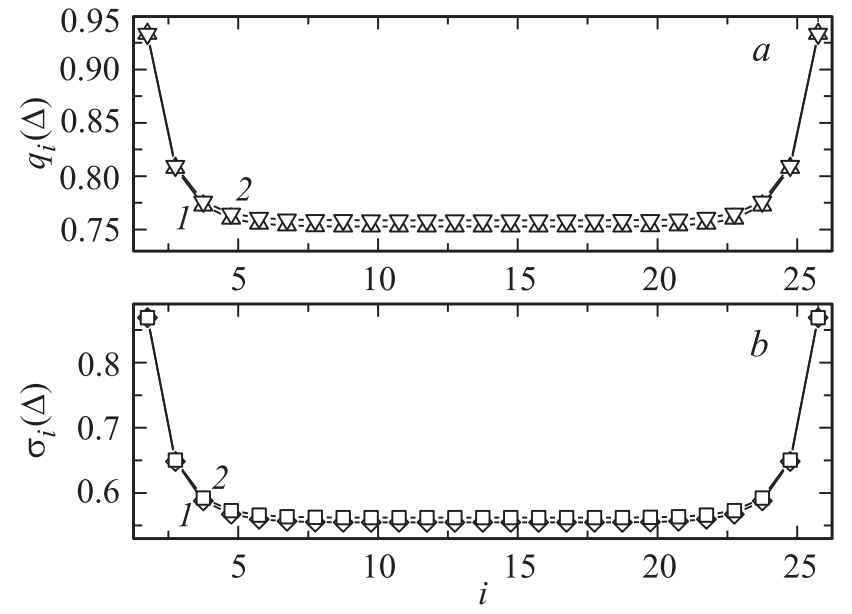

Рис. 1. Профили ориентационного $q_{i}(\Delta)(a)$ и трансляционного $\sigma_{i}(\Delta)(b)$ ПП в зависимости от $i$ по сечению 25-слойной СПСП без учета $(\Delta=0$, кривые 1$)$ и с учетом $(\Delta=0.08$, кривые 2) внешнего электрического поля. Расчеты проведены при безразмерной температуре $\theta=0.67$.

H10F5MOPP [4]. Следует принять во внимание, что величина параметра $V_{0}=2.2 \cdot 10^{-20} \mathrm{~J}$ была рассчитана на основании того факта, что для $\alpha=1.05$, согласно теории Мак-Миллана [11], значение $k_{B} T_{A I}(b) / 0.2202 V_{0}=1.021$. Следует также отметить, что в наших расчетах величина безразмерной температуры $\theta=3 k_{B} T / V_{0}$ варьировалась между $0.6(\sim 318.2 \mathrm{~K})$ и $0.8(\sim 424.3 \mathrm{~K})$. Влияние внешнего электрического поля $\mathbf{E}$ (или $\Delta$ ) как на ориентационные $q_{i}(\Delta)$, так и трансляционные $\sigma_{i}(\Delta)$ ПП в 25-слойной СПСП было исследовано посредством решения системы $2 N$ нелинейных уравнений $(6)-(8)$ и представлено на рис. $1, a$ и $b$. Согласно нашим расчетам, распределение функций $q_{i}(\Delta)$ и $\sigma_{i}(\Delta)$ по сечению 25-слойной СПСП, в отсутствие $(\Delta=0)$ и с учетом $(\Delta=0.08)$ внешнего электрического поля, направленного поперек смектической пленки, характеризуется резким разупорядочением их профилей с ростом $i$, или по мере продвижения со стороны раздела ЖК/воздух к центру пленки. Оба результата были получены при одной и той же безразмерной температуре, равной 0.67 , или $\sim 355.35 \mathrm{~K}$. Располагая профилями $q_{i}(\theta, \Delta)$ и $\sigma_{i}(\theta, \Delta)$ и используя уравнения (5) и (6) [4], можно рассчитать профили как свободной энергии Гельмгольца $f(\theta)$, так и энтропии $s(\theta)$, приходящиеся на одну молекулу смектической пленки. Поскольку наша основная цель описать трансляционную диффузию в процессе утоньшения СПСП, по мере ее нагревания, то мы опустим здесь все детали вычисления $f(\theta)$ и $s(\theta)$, которые приведены в ссылке [4]. Тем не менее, было показано, что в отсутствие электрического поля $(\Delta=0)$ реализуется следующая последовательность утоньшения СПСП: $25 \rightarrow 13 \rightarrow 11 \rightarrow 10 \rightarrow 9 \rightarrow 8 \rightarrow 7 \rightarrow 6 \ldots$, по мере того как температура повышалась, начиная со значения $\theta_{A I}(b) \sim 0.675$. Эта теоретически рассчитанная последовательность утоньшения 25-слойной СПСП, образованной молекулами H10F5MOPP, немного отличается 
Результаты расчета безразмерных температур перехода (layer transition) $\theta_{A I}(N, \Delta)$, соответствующих двум последовательностям

\begin{tabular}{c|c|c|c}
\hline Послойный переход & $\theta_{\mathrm{AI}}(\Delta=0)$ & Послойный переход & $\theta_{\mathrm{AI}}(\Delta=0.08)$ \\
\hline $\mathrm{N}=25 \rightarrow 13$ & $0.678 \quad(\sim 359.6 \mathrm{~K})$ & $\mathrm{N}=25 \rightarrow 14$ & $0.704(\sim 373.4 \mathrm{~K})$ \\
$\mathrm{N}=13 \rightarrow 11$ & $0.697 \quad(\sim 369.7 \mathrm{~K})$ & $\mathrm{N}=14 \rightarrow 12$ & $0.707(\sim 375 \mathrm{~K})$ \\
$\mathrm{N}=11 \rightarrow 10$ & $0.706 \quad(\sim 374.44 \mathrm{~K})$ & $\mathrm{N}=12 \rightarrow 10$ & $0.709(\sim 376.1 \mathrm{~K})$ \\
$\mathrm{N}=10 \rightarrow 9$ & $0.7106(\sim 377 \mathrm{~K})$ & $\mathrm{N}=10 \rightarrow 9$ & $0.713(\sim 378.2 \mathrm{~K})$ \\
$\mathrm{N}=9 \rightarrow 8$ & $0.717 \quad(\sim 380.3 \mathrm{~K})$ & $\mathrm{N}=9 \rightarrow 8$ & $0.716(\sim 379.8 \mathrm{~K})$ \\
$\mathrm{N}=8 \rightarrow 7$ & $0.729(\sim 386.6 \mathrm{~K})$ & $\mathrm{N}=8 \rightarrow 7$ & $0.723(\sim 383.5 \mathrm{~K})$ \\
$\mathrm{N}=7 \rightarrow 6$ & $0.736(\sim 390.3 \mathrm{~K})$ & $\mathrm{N}=7 \rightarrow 6$ & $0.731(\sim 387.7 \mathrm{~K})$ \\
$\mathrm{N}=6 \rightarrow 5$ & $0.743 \quad(\sim 394 \mathrm{~K})$ & $\mathrm{N}=6 \rightarrow 5$ & $0.76(\sim 403.1 \mathrm{~K})$
\end{tabular}

от экспериментально наблюдаемой последовательности $25 \rightarrow 15 \rightarrow 11 \rightarrow 9 \rightarrow 8 \rightarrow 7 \rightarrow 6 \ldots$ [1]. В таблице приведены номера слоев $N$ СПСП, начиная с которых происходит процесс послойного выдавливания внутренних слоев в мениск (первая колонка) и значения температур $\theta_{A I}(N, \Delta=0)$, при которых происходят соответствующие $\Delta=0$ переходы (вторая колонка).

В случае, когда поперек смектической пленки приложено электрическое поле, характеризующееся безразмерным параметром $\Delta=0.08$, последовательность послойных переходов с ростом температуры $\theta>\theta_{A I}(b)$ изменяется. Сначала выдавливается 11 слоев, затем дважды по 2 слоя и далее по одному слою, заканчивая двуслойной пленкой. Номера слоев СПСП, начиная с которых происходит процесс послойного выдавливания внутренних слоев в мениск (третья колонка) и значения температур $\theta_{A I}(N, \Delta=0.08)$, при которых происходят соответствующие $\Delta=0.08$ переходы (четвертая колонка), приведены в таблице. Таким образом, в случае $\Delta=0$, сначала выдавливается 12 слоев, при температуре $\theta_{A I}(N=25, \Delta=0) \sim 0.678(\sim 359) \mathrm{K}$, в то время как в случае $\Delta=0.08$ первый переход утоньшения $25 \rightarrow 14$, реализуется при $\theta_{A I}(N=25, \Delta=0.08) \sim 0.704$ $(\sim 373) \mathrm{K}$, что на $\sim 13.4 \mathrm{~K}$ выше, чем в первом случае $(\Delta=0)$. По мере утоньшения пленки температуры $\theta_{A I}(N, \Delta=0)$ и $\theta_{A I}(N, \Delta=0.08)$ имеют тенденцию к сближению, что и наступает практически при переходе $9 \rightarrow 8$. Дальнейшее утоньшение СПСП приводит к тому, что уже $\theta_{A I}(N=6, \Delta=0) \sim 0.743$, практически на $9 \mathrm{~K}$ ниже, чем $\theta_{A I}(N=6, \Delta=0.08) \sim 0.76$.

Теперь обратимся к расчету диффузионных свойств СПСП в процессе ее утоньшения. Располагая значениями $q_{i}(\theta, \Delta)$ и $\sigma_{i}(\theta, \Delta)$ и используя уравнения (2) и $(11)-(13)$, мы можем рассчитать влияние безразмерной температуры $\theta$ на безразмерный КТД $D_{i, i+1}(\theta, \Delta=0) / D_{N / 2, N / 2+1}(\theta, \Delta=0)$ в зависимости от номера слоя $i$. На рис. 2 представлены результаты расчета $D_{i, i+1}(\theta, \Delta=0) / D_{N / 2, N / 2+1}(\theta, \Delta=0)$ в 25 -слойной СПСП для трех значений безразмерной температуры $\theta=0.67$ (кривая 1), 0.675 (кривая 2), и 0.677 (кривая 3). Все три зависимости характеризуются монотонным ростом значений $D_{i, i+1}(\theta, \Delta=0) / D_{N / 2, N / 2+1}(\theta, \Delta=0)$ по мере удаления от границы раздела ЖК/воздух. В случае параметризации $W_{0} / V_{0}=5$ влияние физической границы раздела простирается на 8 слоев с каждой стороны

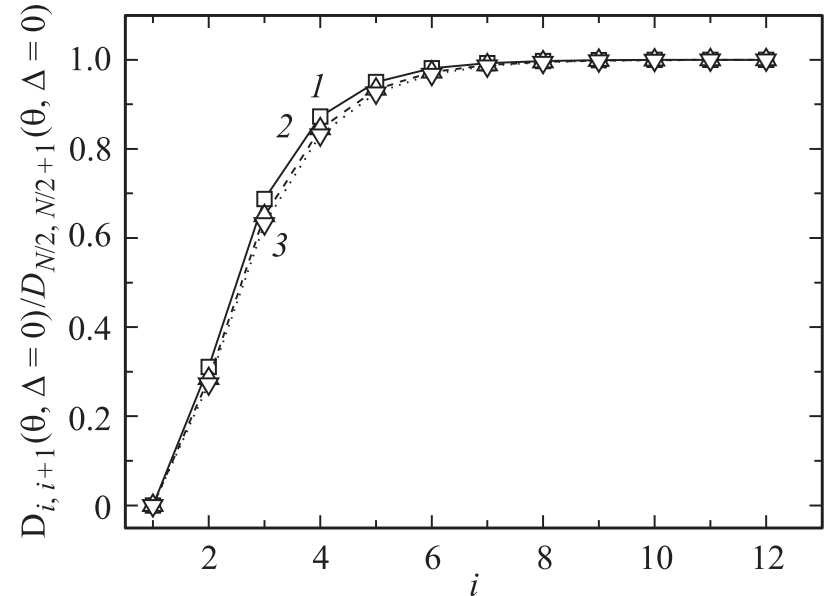

Рис. 2. Профили безразмерного КТД $D_{i, i+1}(\theta, \Delta=0) /$ $D_{N / 2, N / 2+1}(\theta, \Delta=0)$ в зависимости от $i$ по сечению 25 -слойной СПСП без учета $(\Delta=0)$ внешнего электрического поля, для трех значений безразмерной температуры: $\theta=0.67$ (кривая 1 ), $\theta=0.675$ (кривая 2) и $\theta=0.677$ (кривая 3) соответственно.

смектической пленки, в то время как вблизи ограничивающей поверхности безразмерный КТД обращается в 0. Это свидетельствует о том, что поверхностное натяжение не только подавляет термофлуктуации в приповерхностных слоях, но и полностью подавляет трансляционную диффузию молекул из СПСП в воздух. На рис. 3 представлены результаты расчета профилей $D_{i, i+1}(\theta, \Delta=0) / D_{N / 2, N / 2+1}(\theta, \Delta=0) \quad$ в $\quad$ зависимости от $i$ для случая смектической пленки в процессе ее послойного утоньшения, с последующим выдавливанием внутренних слоев в мениск. Вначале, для случая $\Delta=0$, рассчитано распределение $D_{i, i+1}(N=25, \theta=0.68) /$ $D_{N / 2, N / 2+1}(N=25, \theta=0.68)$ по сечению 25-слойной смектической пленки, при значении безразмерной температуры $\theta=0.68(\sim 360.7 \mathrm{~K}) \quad$ (см. рис. 3, кривая 1). Затем, такая же зависимость получена для случая 13-слойной смектической пленки, при температуре $\theta=0.69(\sim 366) \mathrm{K}$ (см. рис. 3, кривая 2). Далее следует 11-слойная пленка, при температуре $\theta=0.70(\sim 371.3) \mathrm{K}$ (см. рис. 3, кривая 3), и, наконец, 10-слойная смектическая пленка, при температуре $\theta=0.71(\sim 376.6) \mathrm{K}$ (см. рис. 3, кривая 4). Во всех этих четырех случаях поверхностное натяжение 


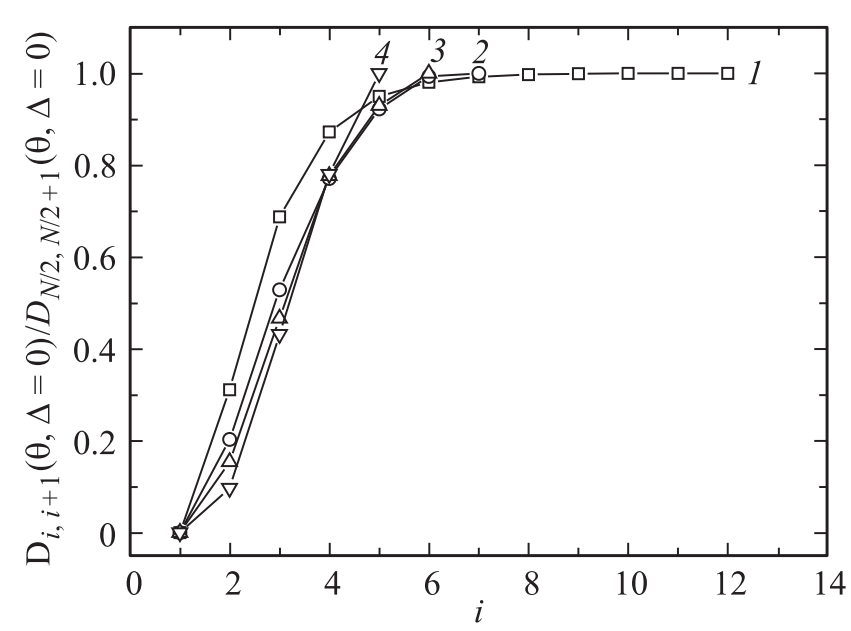

Рис. 3. Зависимость безразмерного КТД $D_{i, i+1}(\theta, \Delta=0) /$ $D_{N / 2, N / 2+1}(\theta, \Delta=0)$ от номера слоя $i$ в процессе послойного утоньшения СПСП. Кривая 1 соответствует 25-слойной, кривая $2-13$-слойной, кривая $3-11$-слойной, и кривая $4-10$-слойной СПСП. Во всех случаях $\Delta=0$.

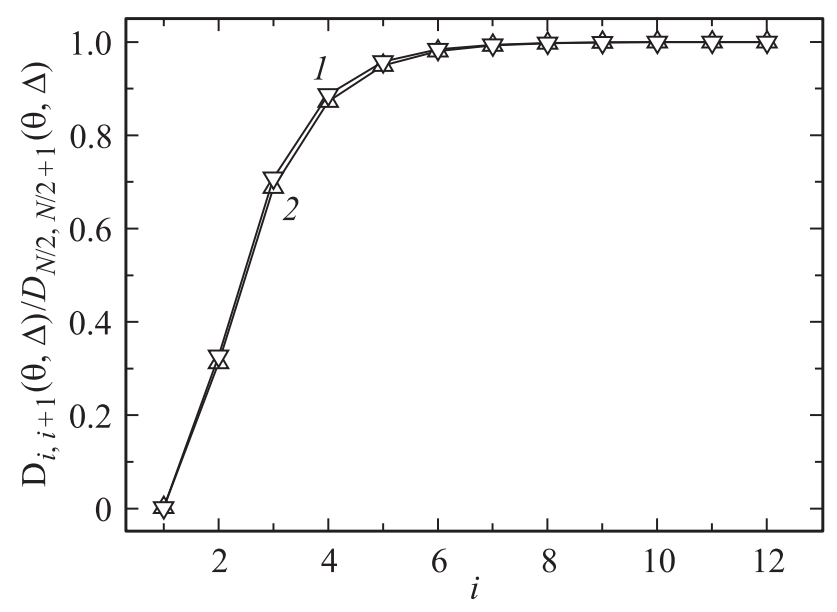

Рис. 4. Влияние величины внешнего электрического поля $\Delta$ на распределение КТД $D_{i, i+1}(\theta, \Delta) / D_{N / 2, N / 2+1}(\theta, \Delta)$ по сечению 25-слойной частично флуорированной H10F5MOPP смектической пленки: Кривая 1 расчитана для случая, когда электрическое поле отсутствует $(\Delta=0)$, в то время как кривая 2 рассчитана для случая, когда внешнее электрическое поле $\Delta=0.08$ приложено поперек смектических слоев.

полностью подавляет трансляционную диффузию из СПСП в воздух. Результаты расчета влияния электрического поля $(\Delta=0.08)$, направленного поперек смектических слоев СПСП на характер профиля $D_{i, i+1}(\theta=0.67, \Delta=0.08) / D_{N / 2, N / 2+1}(\theta=0.67, \Delta=0.08)$ в 25-слойной СПСП, при температуре $\theta=0.67$, представлены на рис. 4 (кривая 2). Здесь, как и в случае отсутствия электрического поля $(\Delta=0)$ (рис. 4, кривая 1 ), поверхностное натяжение полностью подавляет трансляционную диффузию из СПСП в воздух, а само электрическое поле слабо влияет на характер зависимости КТД от расстояния от границы раздела ЖК-фаза/воздух.
Такое поведение КТД объясняется тем, что величина потенциального барьера $\Delta \Phi_{1,2}$, соответствующая граничному слою, значительно превосходит величины потенциальных барьеров $\Delta \Phi_{i, i+1}(i=2, \ldots, N / 2)$, соответствующих внутренним слоям смектической пленки. Это достигается благодаря более сильному приповерхностному упорядочению молекул по сравнению с тем разупорядочением, что наблюдается в объеме СПСП (см. рис. 1). Следует отметить, что наши исследования касаются только лишь трансляционной диффузии, направленной поперек смектических слоев (вдоль направления директора), поскольку все величины необходимые для расчета КТД, были получены с помощью обобщенной среднеполевой модели, которая учитывает не только анизотропные взаимодействия между ближайшими соседями молекул, образующих СПСП, но и стабилизирующее влияние границы раздела смектик/воздух.

Для того чтобы вычислить размерную величину КТД

$$
D_{\|}^{b}(\mathrm{~N}) \equiv D_{b}(\mathrm{~N})=\frac{k_{\mathrm{B}} T}{m} \int_{0}^{\infty} f(t) d t
$$

в объеме $N$-слойной смектической пленки, необходимо располагать значениями автокорреляционной функции импульса $\mathbf{p}(t)$ (или скорости $\mathbf{v}(t))$

$$
f(t)=\langle\mathbf{p}(0) \cdot \mathbf{p}(t)\rangle /\langle\mathbf{p}(0) \cdot \mathbf{p}(0)\rangle .
$$

Здесь $\langle(\ldots)\rangle-$ среднее по равновесному ансамблю [12], a $m$ - масса молекулы. Строгий расчет автокорреляционной функции $f(t)$ представляет собой сложную задачу. В дальнейшем мы будем следовать результатам работы [13], где предполагается, что $f(t)$ может быть представлена в виде функции, зависящей от двух параметров $\alpha$ и $\delta$ как

$$
f(t)=\cos (\alpha \delta t) / \cosh (\alpha t) .
$$

Здесь размерный параметр $\alpha$ [в единицах $\left.s^{-1}\right]$ определяет скорость затухания $\lim _{t \rightarrow \infty} f(t, \alpha) \rightarrow 0$, в то время как безразмерный параметр $\delta$ определяют частоту колебаний $f(t)$ в пределах шкалы времени, которую задает параметр $\alpha$. Величины этих параметров связаны с импульсом и его производными следующими соотношениями: $\alpha^{2}=3(C-1) \frac{\left\langle\dot{p}^{2}\right\rangle}{12\left\langle p^{2}\right\rangle}$ и $\delta^{2}=\frac{5-C}{C-1}$, в то время как величина $C$ равна $\left\langle p^{2}\right\rangle \frac{\left\langle\ddot{p}^{2}\right\rangle}{\left\langle\dot{p}^{2}\right\rangle^{2}}$. Поскольку в случае смектической фазы одночастичная функция Гамильтона имеет вид $H(z)=\frac{p^{2}}{2 m}+\Phi(z)$, где $\Phi(z)$ - величина потенциального барьера возникающего между смектическими слоями, то $\dot{p}=-\frac{\partial H}{\partial z}=-\Phi_{, z}(z)$. Таким образом, величины $\left\langle\dot{p}^{2}\right\rangle$ и $\left\langle\ddot{p}^{2}\right\rangle$ могут быть записаны в виде $\left\langle\dot{p}^{2}\right\rangle=k_{B} T\left\langle\Phi_{, z z}(z)\right\rangle$ и $\left\langle\ddot{p}^{2}\right\rangle=\frac{k_{B} T}{m}\left\langle\Phi_{, z z}^{2}(z)\right\rangle$ соответственно. Здесь

$$
\langle(\ldots)\rangle=\int_{0}^{1} d z(\ldots) \exp \left[-\frac{\Phi(z)}{k_{B} T}\right] / \int_{0}^{1} d z \exp \left[-\frac{\Phi(z)}{k_{B} T}\right]
$$

среднее по ансамблю, а $\Phi_{, z}(z)=\frac{\partial \Phi(z)}{\partial z} \quad$ и $\Phi_{, z z}(z)=\frac{\partial^{2} \Phi(z)}{\partial z^{2}}$ - первая и вторая производные по- 
тенциала $\Phi$ по пространственной переменной $z$ соответственно. С учетом вышесказанного, выражения для $C$ и $\alpha^{2}$ принимают вид

$$
C=3 \frac{\left[\int_{0}^{1} d z g_{, z z}^{2}(z) G(z)\right]\left[\int_{0}^{1} d z G(z)\right]}{\left[\int_{0}^{1} d z g_{, z z}(z) G(z)\right]^{2}},
$$

$$
\alpha^{2}=\frac{k_{B} T}{12 m d^{2}}(C-1) \frac{\int_{0}^{1} d z g_{, z z}(z) G(z)}{\int_{0}^{1} d z G(z)} .
$$

Здесь функция $g(z)=\Phi(z) / k_{B} T=-\frac{1}{\theta}[a+b \cos (2 \pi z)]$, в то время как функция $G(z)=\exp [-g(z)]$, а коэффициенты $a=q_{N / 2}+2 q_{N / 2-1}+\Delta\left(q_{N / 2}+1\right)$ и $b=\sigma_{N / 2}$ $+2 \sigma_{N / 2-1}$ зависят от ориентационных и трансляционных ПП, соответствующих объемным значениям смектической пленки.

Принимая все это во внимание, выражение для КТД в объеме смектической пленки перепишем в виде [13]

$$
D_{b}(\mathrm{~N})=\frac{k_{B} T}{m} \frac{\pi}{\alpha} \cosh ^{-1}\left(\frac{\pi \delta}{2}\right) .
$$

Результаты расчета размерной величины КТД $D_{b}(N=25)$, при температуре $T=367 \mathrm{~K} \quad(\theta=0.67)$, для 25-слойной СПСП, образованной молекулами H10F5МОРР, показали, что $D_{b}(N=25) \sim 6 \mu \mathrm{m}^{2} / \mathrm{s}$, что близко к значению КТД $D_{b}(N=25) \sim 3 \mu \mathrm{m}^{2} / \mathrm{s}$, полученному методами флюорисцентной спектроскопии $[14,15]$, в объеме 25-слойной смектической пленки, образованной молекулами 4-октил-4'-цианобифинила. Результаты расчета размерных значений КТД в объеме СПСП, образованной молекулами H10F5MOPP, в процессе еe утоньшения дали следующий результат: $\quad D_{b}(N=13) \sim 6.3, \quad D_{b}(N=11) \sim 6.7 \quad$ и $D_{b}(N=10) \sim 7.2 \mu \mathrm{m}^{2} / \mathrm{s}$ соответственно.

Таким образом, следует отметить, что величина КТД в объеме СПСП монотонно возрастает по мере ее утоньшения. Физически это оправдано, поскольку процесс утоньшения СПСП обусловлен ростом температуры в объеме смектической пленки, а это, в свою очередь, ведет к уменьшению величины потенциального барьера, разделяющего смектические слои в объеме ЖК-пленки.

\section{4. Заключение}

Предложена молекулярная модель трансляционной диффузии, основанная на теории случайных блужданий и позволяющая рассчитать коэффициент трансляционной диффузии (КТД), направленный поперек смектических слоев (вдоль направления директора), при том что все необходимые для расчета КТД величины были получены в рамках обобщенной среднеполевой модели, учитывающей не только анизотропные взаимодействия между ближайшими соседями молекул, образующих свободно подвешенные смектические пленки (СПСП), но и стабилизирующее влияние границы раздела смектик/воздух.
В случае смектической пленки процесс трансляционной диффузии вдоль направления директора подразумевает скачок диффундирующей молекулы через потенциальный барьер, разделяющий смектические слои. При этом величины потенциальных барьеров, разделяющих смектические слои многослойных СПСП, являются функциями ориентационных $q_{i}$ и трансляционных $\sigma_{i}$ параметров порядка (ПП), не только выбранного смектического слоя, но и его ближайших соседей. Основываясь на поведении свободной энергии Гельмгольца, соответствующей 25-слойной СПСП, образованной молекулами 5-n-алкил-2-(4-n-(перфлуороалкил-метиленеокси)) пентила (H10F5MOPP), было показано, что по мере нагревания смектической пленки, начиная с температуры выше температуры фазового перехода SmA-I, в объеме ЖК-фазы $T_{A I}(\mathrm{~b})$ начинается процесс послойного выдавливания внутренних слоев пленки в мениск. При этом реализуется следующая последовательность утоньшения СПСП: $25 \rightarrow 13 \rightarrow 11 \rightarrow 10 \rightarrow 9 \rightarrow 8 \rightarrow 7 \rightarrow 6 \ldots$. Вычисления также показали, что по мере роста температуры процессу выдавливания определенного числа слоев из СПСП в мениск предшествует процесс внутреннего разупорядочения одного или нескольких слоев, что выражается в резком уменьшении величин $q_{i}$ и $\sigma_{i}$ соответствующих выдавливаемым слоям. При этом температура $T_{A I}(N)$, при которой начинается процесс послойного выдавливания в мениск одного или нескольких слоев, играет роль температуры плавления $N$-слойной пленки. Возникающая при этом пространственная неоднородность ПП по сечению СПСП ведет к тому, что поверхностное натяжение на границе ЖК/воздух не только подавляет термофлуктуации в приповерхностных слоях, но и полностью подавляет трансляционную диффузию молекул из СПСП в воздух. Результаты расчетов также показали, что величина внешнего электрического поля $\mathbf{E}$, приложенного поперек смектических слоев, больше влияет на термодинамические, чем на структурные и диффузионные свойства СПСП. Так, было показано, что профили как ориентационного $q_{i}$, так и трансляционного $\sigma_{i}$ ПП с ростом номера смектического слоя $i$, или по мере удаления от границы раздела ЖК/воздух к центру пленки, слабо зависят от величины безразмерного параметра $\Delta$, ответственного за воздействие электрического поля на смектические слои. В то же время, наличие сильного электрического поля $\Delta=0.08\left(E \sim 2 \times 10^{-2} C / m^{2}\right)$ ведет к количественному изменению процесса утоньшения 25-слойной СПСП, образованной молекулами H10F5MOPР, при температуpe выше, чем $T_{A I}(b)$. Было показано, что при воздействии электрического поля $\Delta=0.08$ реализуется другая, чем в случае $\Delta=0$, последовательность утоньшения СПСП: $25 \rightarrow 14 \rightarrow 12 \rightarrow 10 \rightarrow 9 \rightarrow 8 \rightarrow 7 \rightarrow 6 \ldots$. Результаты расчета размерных величин КТД в объеме СПСП, образованной молекулами H10F5MOPР, в процессе ее утоньшения показали, что величина КТД монотонно возрастает по мере утоньшения смектической пленки.

Принимая во внимание тот факт, что результаты расчета КТД в процессе послойного утоньшения СПСП 
как вблизи границы раздела ЖК/воздух, так и в объеме смектической пленки физически обоснованы, а расчетные данные близки к экспериментально полученным значениям КТД, можно сделать заключение, что предложенная молекулярная модель диффузии в тонких смектических пленках может быть использована для описания диффузионных процессов в других слоистых молекулярных системах, например в биологических мембранах или нерастворимых ламинарных слоях на границе раздела вода/воздух.

\section{Список литературы}

[1] T. Stoebe, P. Mach, C.C. Huang. Phys. Rev. Lett. 73, 1384 (1994).

[2] W.H. de Jeu, B.I. Ostrovskii, A.N. Shalaginov. Rev. Mod. Phys. 75, 181 (2003).

[3] Дж. Займан. Принципы теории твердого тела. Мир, M.(1974). $478 \mathrm{c}$.

[4] A.V. Zakharov, D.E. Sullivan. Phys. Rev. E 82, 041704 (2010).

[5] M. Veum, E. Kutschera, N. Voshell, S.T. Wang, S.L. Wang, H.T. Nguyen, C.C. Huang. Phys. Rev. E 71, 020701(R) (2005).

[6] A.V. Zakharov, A.A. Vakulenko. Phys. Rev. E 86, 031701 (2012).

[7] P.G. de Gennes, J. Prost. The physics of liquid crystals. Oxford Univ. Press, Oxford (1995). 400 p.

[8] G. Moro, P.L. Nordio, U. Segre. Chem. Phys. Lett. 105, 440 (1984).

[9] A.V. Zakharov, I. S̀liwa. J. Chem. Phys. 140, 124705 (2014).

[10] H.R. Glyde. Rev. Mod. Phys. 39, 373 (1967).

[11] W.L. McMillan. Phys. Rev. A 4, 1238 (1971).

[12] J.G. Kirkwood. J. Chem. Phys. 14, 180 (1946).

[13] D.C. Douglass. J. Chem. Phys. 35, 81 (1961).

[14] B. Schultz, D. Tauber, J. Schuster, T. Baumgartel, C. von Borczyskowski. Soft Matter 7, 7431 (2011).

[15] B. Schultz, M.G. Mazza, C. Bahr. Phys. Rev. E 90, 040501(R) (2014). 